\title{
Enunciación
}

\section{Lectura y escritura en un programa formador de maestros para la infancia}

\author{
Reading and writing in an early childhood teacher training program \\ Ingrit Gutiérrez Vega*
}

\section{Resumen}

Este artículo presenta los resultados de la primera fase de la investigación, cuyos objetivos se centran en describir los niveles de lectura y escritura de los estudiantes en diferentes momentos de la formación, precisar estos niveles y las características socioculturales. Así, se pregunta: ¿Cuál es el nivel de lectura y escritura de los estudiantes de Pedagogía Infantil de la Universidad de los Llanos? Para esto, se ubica en la investigación-acción y desde allí se avanza, en la primera fase, a un estudio descriptivo. Se aplicaron dos instrumentos, una prueba para identificar el nivel de lectura y escritura de los estudiantes y una encuesta que arroja algunas comprensiones de estos niveles en la relación sociocultural. La investigación identifica un nivel bajo de lectura y escritura, que coincide con las Pruebas Saber 11 y la institucional de carácter diagnóstico denominada procesos comunicativos, nivel que no es mejorado durante los nueve semestres de formación. Lo anterior indica que al no alcanzar los requerimientos mínimos de lectura y escritura se les dificulta a los estudiantes apropiar los textos sugeridos por sus maestros, desarrollar discursos con posturas críticas y escribir de forma coherente y con estilo propio.

Palabras clave: niveles de literacidad, desempeño académico, argumentación, pragmadialéctica.

\begin{abstract}
This article presents the results of the first phase of the investigation, whose objectives are focused on describing the levels of reading and writing of the students at different moments of the training, specifying these levels and the sociocultural characteristics. Thus, questions are asked about what is the level of reading and writing of the students of Bachelor's Degree in Childhood Pedagogy at the University of Los Llanos? For this, it is located in action research and from there it advances, in the first phase, to a descriptive study. Two instruments were applied, a test to identify the level of reading and writing of the students and a survey that shows some understandings of these levels in the sociocultural relationship. The research identifies a low level of reading and writing, which coincides with the Saber 11 tests and the institutional diagnostic test called "communication processes", a level that is not improved during the nine semesters of training. The aforementioned indicates that when the minimum reading and writing requirements are not reached, it is difficult for students to appropriate the texts suggested by their teachers, develop speeches with critical positions and write in a coherent way and with their own style.
\end{abstract}

Keywords: literacy levels, academic performance, argumentation, dialect pragmatic.

Doctora en Ciencias Sociales, Niñez y Juventud de la Universidad de Manizales y el Centro Internacional de Educación y Desarrollo Humano (Cinde). Profesora del programa de Educación Infantil de la Universidad de los Llanos. Correo electrónico: igutierrez@unillanos.edu.co

Cómo citar: Gutiérrez Vega, I. (2020). Lectura y escritura en un programa formador de maestros para la infancia. Enunciación, 25(2), 247-261. https://doi. org/10.14483/22486798.16048

Artículo recibido: 16 de marzo de 2020; aprobado: 26 de agosto de 2020 


\section{Introducción}

Asumir que los estudiantes universitarios llegan a su formación profesional con las herramientas necesarias que les permiten construir los conocimientos específicos de un curso, área o profesión, es una afirmación cuestionable. Carlino (2005); López y Arciniegas (2003), y Salazar et al. (2015) señalan que, si bien los estudiantes luego de su primer año de formación universitaria desarroIlan estrategias que les facilitan su encuentro con la educación superior, estas son insuficientes para construir conocimientos de manera profunda en sus disciplinas obtenidas de la lectura de textos. Dadas las dificultades con las que ingresan a la universidad los estudiantes, en especial respecto a la comprensión de textos y a la lectura intertextual, no se cumple con los objetivos: leer para aprender, leer para pensar, leer para conocer. Lo anterior en el entendido de que leer demanda, además del desarrollo de los procesos cognitivos, "anticipar lo que dirá un escrito, aportar nuestros conocimientos previos, hacer hipótesis y verificarlas, elaborar inferencias para comprender lo que se lee, lo que se sugiere, construir un significado, etcétera" (Cassany, 2006, p. 21), es adquirir conocimientos socioculturales particulares de cada práctica concreta de lectoescritura, puesto que "leer es un verbo transitivo" (Cassany, 2004, p. 6) ${ }^{1}$.

Cuando se presenta la necesidad de escribir textos para plantear discusiones relativas a la disciplina, al diálogo entre disciplinas o para expresar y estructurar un pensamiento crítico, Lozano (2015) encuentra que los textos se organizan con un fin instrumentalista en el que se obvia la transformación del conocimiento. Además, como

1 Para Cassany (2006) la lectura se mueve en tres concepciones: la primera, la lingüística, que implica "recuperar el valor semántico de cada palabra y relacionarlo con el de las palabras anteriores y posteriores. El contenido surge de la suma del significado de todos los vocablos y oraciones" (p. 25). La segunda es la psicolingüística en donde se precisa que el significado es una relación entre el texto y la mente del lector. La tercera concepción es la sociocultural, en palabras de Cassany (2006): "leer no es solo un proceso psicobiológico realizado con unidades lingüísticas y capacidades cognitivas. También es una práctica cultural insertada en una comunidad particular, que posee una historia, una tradición, unos hábitos y unas prácticas comunicativas especiales" (p. 38). precisa Carlino (2005), "los escritos de los estudiantes generalmente expresan la narrativa del pensamiento de los autores que leen y no se anticipan a las necesidades informativas de sus destinatarios" (p. 323).

Así las cosas, en la investigación se retoma a Cassany (1999) quien prefiere utilizar el concepto composición escrita, puesto que alude a la construcción del texto, lo cual exige: dominio del código escrito, elaboración de la información (selección, resumen, ampliación, modificación, entre otros), generación de conocimiento nuevo, ser un instrumento de actuación social, además de cumplir con una función performativa y lúdica² (p. 49).

$\mathrm{Al}$ respecto, se encuentran algunas investigaciones que se preguntan por los niveles de lectura y escritura de los futuros profesionales, entre las que está la desarrollada por Castañeda y Henao (2005) en la Facultad de Economía de la Universidad de Antioquia, que compara los resultados del curso Español trazado con un enfoque tradicional, frente a uno asumido según la literacidad y el análisis crítico del discurso, demostrando la ventaja que ofrece este último para el desarrollo de habilidades en los estudiantes. Otra investigación a propósito de la lectura y la escritura en los programas de pregrado es la desarrollada en la Universidad de Medellín por Henao y Toro (2008), quienes ratifican la necesidad de la comprensión lectora y la producción escrita según la literacidad y el análisis crítico del discurso.

En la misma línea, es posible identificar la investigación realizada en la Institución Universitaria de Envigado, por Londoño, Henao, Frías y Marín (2010), que señala la falta de estrategias

2 En Cassany (1999) las funciones de la escritura se clasifican en intralingüística, interligüística y lúdica. La primera con funciones para el mismo escritor, como: registrativa (superación de la volatilidad de la oralidad o la fragilidad de la memoria), manipulativa (moldeamiento de las actividades diarias y reformulación de lo enunciado) y epistémica (generación de opiniones que no se tenían antes de la actividad escritora, es decir, crear y aprender conocimientos nuevos). En lo que respecta a la interligüística, la escritura es un canal de actuación social, puesto que se escribe para otros, así que tiene una función comunicativa (demanda el dominio de los usos descontextualizado del lenguaje) y certificativa (performativa). Finalmente está la función lúdica de la escritura, que le permite su uso como entretenimiento, belleza, ironía, entre otras. 
adecuadas en los estudiantes para resumir, la escaza fuerza argumentativa, la falta de coherencia y la dificultad para encontrar sentidos implícitos en los textos. En este mismo contexto institucional, Londoño (2013) trabaja el análisis sociolingüístico de los niveles de literacidad en jóvenes y encuentra una relación directa entre promedios bajos de literacidad y estrato social bajo, y precisa la necesidad de implementar estrategias pedagógicas para superar deficiencias en la lectura y la escritura.

Estas observaciones condujeron a formular la pregunta de investigación: ¿Cuál es el nivel de lectura y escritura de los estudiantes del programa de Pedagogía Infantil de la Universidad de los Llanos? Este interrogante coincide con las indagaciones de Serrano (2014), y de Moyano y Giudice (2016). Para responderla, fue necesario buscar una prueba que permitiera identificar el nivel alcanzado por los estudiantes en lectura y escritura (Henao, Londoño, Frías y Castañeda, 2011; Londoño y Ospina, 2018). Igualmente, se utilizó una encuesta sobre aspectos socioculturales (Calderón y Quijano, 2010; Londoño, 2016) que en un diálogo con la prueba arrojó algunas comprensiones (Rodríguez, 2005; Hussein, 2015). Los dos instrumentos permitieron recoger la información que sirvió de corpus investigativo (Stubbs, 2014; Hyland, 2015).

\section{Metodología}

El método es cualitativo (Denzin y Lincoln, 2012) y adopta la perspectiva de la investigación-acción (Lewin, 1973), puesto que, en el contexto educativo universitario, la actividad del investigador se centra en identificar situaciones que afectan la enseñanza o el aprendizaje. Respecto a los niveles de lectura y escritura, o como lo plantea Quitián (2017) "en razón a favorecer innovaciones y transformaciones en las prácticas de enseñanza de los profesores, las cuales afectan los aprendizajes de los estudiantes" (p. 146). A partir de esta premisa, se estructura una fase reflexiva que Ileva a la consolidación de una propuesta que es implementada, evaluada y produce cambios significativos ante la situación problema. En esta forma, y siguiendo a Lewin (1973), la investigación-acción es cíclica y comprende pasos como la reflexión, la acción y la evaluación de la acción.

En este sentido, el primer paso fue la reflexión sobre el problema identificado, a partir de los datos recogidos (corpus investigativo) que permitieran precisar el nivel de lectura y escritura de los estudiantes, para lo cual fue indispensable definir dos instrumentos, una prueba y una encuesta. Los datos recogidos con la prueba se analizaron a la luz de una rúbrica que refleja los requerimientos y aspectos a evaluar (en cada aspecto se definió un criterio y un puntaje que va de 0,0 a 2,0 y el mínimo 0,0). La encuesta se asumió desde lo sociolingüístico y preguntó por aspectos socioculturales, información académica, hábitos de lectura y escritura y la experiencia formativa, respuestas que se pusieron en diálogo con lo encontrado en la prueba. Si bien el posicionamiento de la investigación es cualitativo, fue indispensable el uso de técnicas estadísticas (promedio) como instrumento para describir y reflejar los datos, y desde allí, avanzar en el análisis.

El segundo paso corresponde a la acción, que implica la formulación de la propuesta pedagógica con la que se pretende potencializar los niveles de lectura y escritura en los estudiantes del programa, partiendo de las debilidades y las fortalezas detectadas y en la que confluyen la teoría y la práctica, para producir un saber contextualizado que permita transformar la didáctica.

El tercer paso implica la validación de la propuesta pedagógica, que se desarrollará con los estudiantes con los que se realizó la prueba inicial y que todavía se encuentren activos. Es de aclarar, que el segundo y tercer paso forman parte de la fase 2 del proyecto de investigación a desarrollarse a finales de 2020.

De esta forma, se partió de la revisión bibliográfica de los requerimientos de literacidad exigidos a un joven universitario según Henao et al. (2011), 
lo que permitió identificar los criterios de evaluación y los postulados teóricos que estructuraran el proceso. Estos requerimientos son: resumir (Van Dijk, 1991; Henao y Castañeda, 2002; Gastel y Day, 2016; Eco, 1985), leer críticamente (Van Dijk, 1999; Ferreiro, 2003; Cassany, 2006; Cardona y Londoño, 2016; Wallace y Wray, 2016), argumentar (Van Eemeren y Grootendorst, 2006; Moreno, 2012; Betancourt y Frías, 2015; Newell, Bloome y Hirvela, 2015) y citar y referenciar (Ossa, 2003; Mayor, 2006; APA, 2014; Sánchez, 2015).

En las producciones de los estudiantes deben tenerse en cuenta todos estos criterios de evaluación, para lo cual se diseñó una prueba con tres requerimientos como a continuación se señala:

Resumir: empleo de macrorreglas en la elaboración del resumen (Van Dijk, 1991; Almeida, Matías y Fernández, 2010), cohesión (Cassany, 1995; Louwerse, 2004), coherencia (Beaugrande y Dressler, 1997; McNamara, 2004), compresión de los significados en su elaboración (Braslavsky, 2005; Cassany, 2006), uso de los marcadores discursivos (Briz y Pons, 2010; Borreguero y López, 2011) y distanciamiento del texto (Zavala, 1989; Espinosa, 2017) en la elaboración del resumen.

Para realizar una lectura crítica, se tuvieron en cuenta: la explicación sobre los procesos de la lectura crítica (Calderón y Quijano, 2010; Stubbs, 2014), ortografía (Camps et al., 2011; Londoño y Ospina, 2018), puntuación ${ }^{3}$ (Cassany, 1995; Cuetos, Molina, Suárez y Llenderrozas, 2017), leer críticamente (Ferreiro, 2003; Cassany, 2006), estructura de los textos (Beaugrande y Dressler, 1997; Londoño, 2015) y distanciamiento del texto (Betancourt y Frías, 2015; Henao et al., 2011).

Respecto a la argumentación, se precisaron aspectos como planteamiento de la tesis (Beaugrande y Dressler, 1997; McNamara, 2004), construcción

3 Si bien la ortografía y la puntuación no están conectadas directamente con los procesos de comprensión textual, lo están con los de producción teniendo en cuenta que permiten la calidad del texto en la parte estilística. En la perspectiva de la lingüística textual (que se asume en esta investigación) se evalúan otros aspectos: cohesión, coherencia, discursividad y la forma como se construyen los argumentos. de los argumentos (Ferreiro, 2003; Hyland, 2015), uso de las citas como garantes (Henao y Castañeda, 2002; Moreno, 2012), citar correctamente y referenciar fuentes utilizadas (Ossa, 2003; Mayor, 2006), utilizar las posiciones de otros autores para argumentar o contraargumentar (Newell, Bloome y Hirvela, 2015; Gastel y Day, 2016), uso de falacias argumentativas (Van Eemeren et al., 2014) y argumentar la tesis de forma coherente, cohesionada y clara (Londoño, 2016; Wallace y Wray, 2016).

Definidos los aspectos de la evaluación para cada requerimiento (18 en total), que conformaron la prueba, se dio paso a la selección de dos lecturas, y se escogieron dos textos: el primero fue el artículo "Si yo gobernara el mundo", de Steven Pinker (2011), respecto al cual se les pidió a los estudiantes que hicieran un resumen ${ }^{4}$ y enunciaran la técnica utilizada. Para la segunda lectura, se eligió el artículo publicado en El Espectador, titulado "Lecto-escritura", de José Fernando Isaza (2012)5, se les invitó a realizar una lectura crítica y a precisar qué es una lectura crítica ${ }^{6}$.

La prueba se diligenció con estudiantes del programa de Pedagogía Infantil que tomaban cursos en los semestres I, IV y VII. Se seleccionó una muestra aleatoria por semestre equivalente al $30 \%$, de modo que, en total, se trabajó con 35 estudiantes (14 de primer semestre, 9 de cuarto y 12 de séptimo). Al principio del proyecto, el programa de Pedagogía Infantil contaba con 306 estudiantes, 46 en primer semestre, 29 en cuarto y 40 en séptimo. Es preciso aclarar que los semestres se seleccionaron de forma intencional (Stubbs, 2014), teniendo en

4 En el ejercicio realizado por los estudiantes se evaluó (por parte de dos profesores) el empleo de las macrorreglas, para esto se diseñó una rúbrica en la que además se tuvo en cuenta: la cohesión, la coherencia, la comprensión de significados, el uso de marcadores discursivos y el distanciamiento del texto.

5 Se seleccionaron estas pruebas puesto que ya habían sido realizadas por Henao, Londoño Frías (2014).

6 Aquí se identifica cómo comprenden los estudiantes la lectura crítica y si lo que creen está ajustado al concepto, lo que se pretende es confirmar las creencias previas con respecto a lo esperado. Lo anterior atendiendo a como lo precisan Torres y Londoño (2016): "La lectura crítica cumple una función importante y trascendental en el proceso de formación universitaria, porque es un concepto que implica la identificación de sentidos e intencionalidad al interior de cualquier tipo de texto [...]. Su propósito es buscar la relación entre el creador, la obra, el contexto y el espectador" (p. 382). 
cuenta la duración del programa y el interés por conocer la evolución de los niveles de lectura y escritura de los estudiantes en su formación.

\section{Resultados}

Evaluada la prueba a partir de los criterios determinados en la rúbrica, se identifica el desempeño de cada grupo como se muestra en la tabla 1.

En el grupo que está iniciando la formación, se observa un bajo desempeño en la elaboración del resumen, cuyo puntaje significativamente menor es el uso de macrorreglas $(0,2)$ y el mayor tiene que ver con el distanciamiento del texto $(1,3)$.
Llama la atención que en este grupo el desarrollo de argumentos es nulo.

En el grupo de cuarto semestre, se observa un mínimo incremento frente al primer semestre en el resumen, y coinciden en que el puntaje mínimo es el uso de reglas, pero muestra un menor puntaje en el distanciamiento del texto $(1,3)$. Un comportamiento parecido tienen los grupos en la lectura crítica en la que ambos alcanzan un promedio de 0,33. Lo mismo sucede con el desempeño de los dos grupos en la argumentación cuyo promedio en el primero es de 0,0 y en el de cuarto de 0,1.

En el grupo de séptimo semestre, se identifica un incremento frente a primer semestre, en un $180 \%$

Tabla 1. Promedio de la prueba por semestres ${ }^{7}$

\begin{tabular}{|c|c|c|c|}
\hline Requerimiento y aspectos evaluados & $\begin{array}{l}\text { Promedio grupo de } \\
\text { primer semestre }\end{array}$ & $\begin{array}{l}\text { Promedio grupo de } \\
\text { cuarto semestre }\end{array}$ & $\begin{array}{l}\text { Promedio grupo de } \\
\text { séptimo semestre }\end{array}$ \\
\hline Resumen & $0,8^{*}$ & $1,0^{*}$ & $1,8^{*}$ \\
\hline Empleo de macrorreglas & 0,2 & 0,7 & 1,8 \\
\hline Cohesión & 1,0 & 1,1 & 1,8 \\
\hline Coherencia & 1,0 & 1,1 & 1,9 \\
\hline Comprensión de significados textuales & 0,4 & 0,9 & 1,9 \\
\hline Marcadores discursivos & 1,1 & 1,2 & 1,4 \\
\hline Distanciamiento del texto & 1,3 & 1,3 & 2,0 \\
\hline Lectura crítica & $0,3^{* *}$ & $0,3^{* *}$ & $1,3^{* *}$ \\
\hline Explicación procesos de lectura crítica & 1,0 & 0.9 & 1.4 \\
\hline Ortografía & 0,1 & 0,1 & 0,6 \\
\hline Puntuación & 0,3 & 0,3 & 1,2 \\
\hline Lectura crítica & 0,1 & 0,2 & 1,7 \\
\hline Estructura del texto & 0,1 & 0,3 & 1,0 \\
\hline Distanciamiento del texto & 0,4 & 0,2 & 1,7 \\
\hline Argumentación & $0,0^{* * *}$ & $0,1^{* * *}$ & $0,4^{* * *}$ \\
\hline Planteamiento de la tesis & 0,0 & 0,3 & 1,0 \\
\hline Construcción de argumentos & 0,0 & 0,2 & 0,8 \\
\hline Uso de citas & 0,0 & 0,0 & 0,0 \\
\hline Citas y referencias & 0,0 & 0,0 & 0,0 \\
\hline Empleo de posiciones de otros autores & 0,0 & 0,0 & 0,6 \\
\hline Falacias & 0,0 & 0,0 & 0,0 \\
\hline
\end{tabular}

* promedio en elaboración del resumen.

Fuente: elaboración propia.

** promedio en lectura crítica.

*** promedio en argumentación. 
en el nivel de desempeño en la elaboración del resumen; sin embargo, no se alcanza el nivel esperado. De igual forma, en la lectura crítica, se incrementa alrededor del $363 \%$. No obstante, no se alcanza el nivel esperado. El nivel de más bajo desempeño se observa en la argumentación que alcanza un nivel medio $(1,0)$.

\section{Elaboración del resumen}

Con el propósito de determinar los criterios que permitieran evaluar cualitativa y cuantitativamente cada uno de los aspectos de cada requerimiento, se precisó lo detallado en la tabla 2.

Respecto a las macrorreglas, en el grupo de primer semestre los estudiantes aplican algunas de ellas. Esto se expresa en el puntaje 0,2, que se incrementa levemente en cuarto semestre a un 0,7 , pues usan la supresión y la generalización, y se observa un intento intuitivo de usar la construcción, pero no se ve apropiada la técnica de macrorreglas. En séptimo semestre, el promedio de los estudiantes es de 1,8; además, se reconoce el uso de la supresión, la generalización y la construcción. Según estos datos, es posible precisar que el nivel con el que ingresan al programa los estudiantes, en este requerimiento, es bajo y los procesos formativos que se dan en la licenciatura favorecen su desarrollo, especialmente en los últimos semestres, pero sin alcanzar el nivel más alto.

Respecto a la cohesión, los textos que construyen los estudiantes de primero y cuarto semestres tienen oraciones largas que no permiten identificar el sentido con facilidad, y esta extensión de las oraciones conduce a dificultades de concordancia de número y género. Lo mismo sucede con el queísmo, el dequeísmo, y la dequefobia, lo que coincide con promedios similares entre los dos grupos $(1,0 \text { y } 1,1 \text {, respectivamente })^{8}$. En los textos

$8 \quad$ Al respecto, si bien Casas (2015) describe el dequeísmo como el resultado de un progreso de la sintaxis castellana dada en unos contextos socioculturales específicos, la literatura alrededor del tema acuerda que el queísmo y el dequeísmo en los textos académicos son considerados una transgresión o incorrección idiomática. Lo mismo sucede con la dequefobia, que es la supresión de la preposición de cuando esta es necesaria (Borreguero y López, 2011; Camps et al., 2011; Serradilla, 2017). producidos por los estudiantes de séptimo semestre, es notoria la reducción de problemas de cohesión que alcanza un promedio de 1,8, cercanos al valor esperado $(2,0)$.

En correspondencia con el nivel de desempeño, respecto a la cohesión de los textos construidos por los estudiantes, está el desarrollo de la coherencia textual, que tiene un promedio de 1,0 en primer semestre, 1,1 en cuarto y 1,8 en séptimo. En la construcción de los escritos se observa la falta de uso de marcadores y de signos de puntuación. En más de la mitad de los estudiantes de primero y de cuarto semestre, se da el distanciamiento del autor en la construcción del resumen, pero no su elaboración estructurada.

En la comprensión de significados, en los estudiantes de primer y cuarto semestres, el promedio de comprensión de significados es cercano $(0,4$ y 0,9 , respectivamente), lo que se expresa en una construcción del resumen en el que se traen párrafos completos del texto sin entender el significado y en la tergiversación las ideas del autor, y en ocasiones se afecta la fidelidad informativa del texto, contrario a lo encontrado en los estudiantes de séptimo semestre, que tienen un promedio de 1,9, cercano al esperado.

Respecto a los marcadores discursivos, los tres grupos en este aspecto tienen promedios cercanos (primer semestre 1,1; cuarto, 1,2; y séptimo, $1,3)$, pues, como se señaló antes, los estudiantes no utilizan con suficiencia los marcadores discursivos, lo que lleva al abuso de adjetivos y sustantivos ${ }^{9}$.

Finalmente, en relación con el distanciamiento del texto, es notorio que este es uno de los aspectos que puntúa más alto en los tres grupos: 1,3 en primero, 1,2 en cuarto y 2,0 en séptimo. Estos últimos reconocen en el ejercicio de reescritura que los planteamientos provienen del texto

9 Para Huguet et al. (2018), en la escritura científica de corte empírico-analítico es necesario ser claros, precisos, breves y concisos; por tanto, predominan las oraciones cortas. No obstante, en las ciencias sociales y humanas, entre ellas la educación, la construcción discursiva responde a otras lógicas como la elaboración de oraciones más extensas y la frecuencia de marcadores discursivos que afiancen los rasgos estilísticos. 
leído, lo que ayuda a reconocer la preeminencia que tiene el texto sobre el lector en el proceso de resumir. En los textos de los estudiantes de primer semestre, se reconoce la voz del autor, pero no tienen habilidades para elaborar el resumen desde la perspectiva sociocognitiva. Puede darse por la influencia (o explicaciones) recibidas en su proceso de formación.

\section{Realización de la lectura crítica}

Los criterios para evaluar los aspectos definidos en este requerimiento se relacionan en la tabla 3.

\section{Explicación de la lectura crítica}

Los estudiantes tienen una idea somera de lo que es la lectura crítica, pero, al intentar definirla, les faltan elementos, aunque hacen un esbozo del concepto. Quienes hacen un ejercicio más cercano a la definición son los estudiantes de séptimo semestre con un promedio de 1,4. Por ejemplo, escriben:

Es la capacidad de comprender un escrito y brindar mi punto de vista sobre el contenido y sobre si estoy de acuerdo o no y por qué. (p. 27. s)

Tabla 2. Criterios de evaluación de la elaboración del resumen

\begin{tabular}{|c|c|c|}
\hline Puntuación & Criterio & Aspecto \\
\hline 2 & Esperado & \multirow{3}{*}{$\begin{array}{l}\text { Empleo de macrorreglas en la elaboración del resumen } \\
\text { Cohesión } \\
\text { Coherencia } \\
\text { Comprensión de significados textuales }\end{array}$} \\
\hline 1 & Aceptable & \\
\hline 0 & Insuficiente & \\
\hline 2 & Entre 5 y 8 & \multirow{3}{*}{ Uso de marcadores discursivos } \\
\hline 1 & Entre 2 y 4 & \\
\hline 0 & 1 o ninguno & \\
\hline 2 & Sí & \multirow{2}{*}{ Se distancia del texto } \\
\hline 0 & No & \\
\hline
\end{tabular}

Fuente: elaboración propia.

Tabla 3. Criterios de evaluación de la lectura crítica

\begin{tabular}{|c|c|c|}
\hline Puntuación & Criterio & Aspecto \\
\hline 2 & Esperado & \multirow{3}{*}{ Explicación y lectura crítica } \\
\hline 1 & Aceptable & \\
\hline 0 & Insuficiente & \\
\hline 2 & 0 errores & \multirow{3}{*}{ Ortografía y puntuación } \\
\hline 1 & De 1 a 5 errores & \\
\hline 0 & Más de 5 errores & \\
\hline 2 & Esperado & \multirow{3}{*}{$\begin{array}{l}\text { - Lectura crítica } \\
\text { - Producción de texto }\end{array}$} \\
\hline 1 & Aceptable & \\
\hline 0 & Insuficiente & \\
\hline 2 & Sí & \multirow{2}{*}{ - Se distancia del texto } \\
\hline 0 & No & \\
\hline
\end{tabular}

Fuente: elaboración propia. 
Es el ejercicio visual y mental que implica a la persona, aparte de leer, generar conocimiento y opiniones sobre lo que se está leyendo, es precisamente eso que indica el nombre, una crítica, una reflexión y puesta en marcha de saberes para dar manifiesto ya sea positivo o negativo, a favor o en contra de que se lee. (p. 32. s)

Quienes presentan una confusión al respecto son los del grupo de cuarto con un promedio de 0,9 , como se muestra en los siguientes apartes:

Es reconocer, analizar e interpretar aquellas ideas que se encuentra en un texto pues consiste en identificar necesariamente ideas que no están a simple vista y que se alcanzan a obtener al momento de sintetizar y analizar. (p. 17. c)

Es analizar o comprender algún texto. (p. 19. c)

Un aspecto en el que tienen menor nivel de desempeño es la ortografía ${ }^{10}$, pues el promedio de primero es 0,1 , en cuarto de 0,0 y en séptimo de 0,6 ; y es interesante que los errores ortográficos en los grupos oscilan entre 2 y 35 , es decir, ninguno de los estudiantes tiene menos de 2 errores.

La puntuación es el segundo aspecto con más bajo promedio, con un comportamiento igual entre los grupos de primero y cuarto $(0,33)$ y un ligero incremento en el promedio de séptimo $(0,6)$.

En la escritura de textos que hacen los estudiantes de primero y cuarto semestres, no se elabora una lectura crítica; puesto que, al intentar distanciarse del autor, sus planteamientos se hacen intrincados, se quedan en la opinión somera sin argumentos y contraargumentos. Se carece claridad sobre la idea del autor, faltan referencias y la citación no es adecuada ${ }^{11}$. Esta situación se supera

10 Ahora, la ortografía por sí sola no indica mayor cosa, es relevante cuando se le asocia a otras prácticas como la lectura o los hábitos lectores. Es decir, si entendemos que cuanto más se lee, mejor es la ortografía por la capacidad de reconocimiento de las palabras, esto indicaría que las prácticas de lectura no son tan frecuentes como lo indican ellos en la encuesta.

11 Esto se contradice con la respuesta que dan los estudiantes al preguntarles en la encuesta si citan fuentes. en los estudiantes de séptimo semestre, según se identifica en el promedio del aspecto, 1,7, y que es coherente con la respuesta que dan en la encuesta sobre a si citan fuentes.

En este grupo, se identifica una producción interesante con elementos que indican la comprensión del punto de vista del autor. Sin embargo, es necesario superar la citación tácita, el uso de garantes y avanzar en la producción argumentativa. En los grupos de primero y cuarto semestres, los niveles de producción son incipientes y se pierde de vista la crítica del autor, lo que se observa en los bajos promedios 0,1 y 0,4 , respectivamente.

En cuanto al distanciamiento del texto, el promedio del grupo de primer semestre es de 0,4 y el de cuarto de 0,2 , lo que permite comprender que no se resguardan las ideas que provienen del autor $y$, por tanto, se quedan en opiniones sin avanzar a una lectura crítica, situación que se supera en gran medida en el grupo de séptimo cuyo promedio se incrementa a 1,6 .

\section{La argumentación}

Para evaluar este requerimiento se precisaron los criterios descritos en la tabla 4.

Sobre la argumentación, la valoración promedio del grupo de primer semestre es de 0,0 y la de segundo 0,1; esto implica que no se encuentra una producción en los estudiantes de los primeros semestres, sus escritos se estructuran con opiniones superficiales que carecen de garantes para apoyar lo dicho y no se distancian de los argumentos del autor y terminan siendo conclusiones apresuradas o afirmaciones falaces, al punto de distorsionar los argumentos del autor. En estos dos grupos, la elaboración de los textos se centra en apreciaciones someras que permiten decir que aún no hay elementos de argumentación.

Respecto al grupo de séptimo semestre, el promedio es de 1,0, en algunos estudiantes se observa 
Tabla 4. Criterios de evaluación de la elaboración de la argumentación

\begin{tabular}{|c|c|c|}
\hline Puntuación & Criterio & Aspecto \\
\hline 2 & Esperado & \multirow{3}{*}{ Planteamiento de la tesis } \\
\hline 1 & Aceptable & \\
\hline 0 & Insuficiente & \\
\hline 2 & Esperado & \multirow{3}{*}{ Construcción de argumentos } \\
\hline 1 & Aceptable & \\
\hline 0 & Insuficiente & \\
\hline 2 & Esperado & \multirow{3}{*}{ Uso de citas como garantes } \\
\hline 1 & Aceptable & \\
\hline 0 & Insuficiente & \\
\hline 2 & Esperado & \multirow{3}{*}{ Cita y referencia } \\
\hline 1 & Aceptable & \\
\hline 0 & Insuficiente & \\
\hline 2 & Esperado & \multirow{3}{*}{ Uso de posiciones de otros autores para argumentar y contraargumentar } \\
\hline 1 & Aceptable & \\
\hline 0 & Insuficiente & \\
\hline 5 & 0 falacias & \multirow{3}{*}{ Omisión de falacias } \\
\hline 1 & De 1 a 2 falacias & \\
\hline 0 & Más de tres falacias & \\
\hline
\end{tabular}

Fuente: elaboración propia.

una producción que implica comprender los argumentos de los autores. La tesis logra plantearse de forma aceptable pero no se desarrolla, incurren en falacias, les hacen falta citas y referencias de otros autores para argumentar y contraargumentar. En general, este grupo alcanza un promedio mayor que los dos anteriores, pero aún falta trabajar la producción argumentativa.

Al analizar los resultados de la prueba aplicada en la investigación frente a los puntajes de la Prueba Saber 11 (lenguaje o lectura crítica) y la conducta de entrada de carácter institucional (procesos comunicativos), se observa que hay una tendencia en el desempeño de los estudiantes. Así, por ejemplo, los estudiantes de cuarto semestre ingresan con el puntaje más bajo, de los tres grupos, en la Prueba Saber 11 y obtienen un puntaje más bajo, frente a los otros dos grupos, en la de procesos comunicativos ${ }^{12}$ y en la correspondiente a la investigación solo alcanzan un incremento de 0,2 en el resumen y de 0,1 en argumentación y ninguno en lectura crítica.

\section{Resultados de la encuesta}

En los resultados de las encuestas se encuentra que el $78,1 \%$ habitan en viviendas de estratos 1 y 2 . El 21,8\% reporta que la formación de la madre es a nivel de básica primaria completa o media completa o educación técnica (las tres opciones tienen el mismo porcentaje). La formación del padre en

\footnotetext{
12 En correspondencia con la información suministrada por las oficinas de Admisiones y el Programa de Retención de la Universidad, el promedio de los puntajes en la Prueba Saber 11 para los grupos de primer, cuarto y séptimo semestres es: 54, 47,3 y 56,8, respectivamente. En la prueba institucional los resultados obtenidos son: 3,38, 2,44 y 3,49 (rango de evaluación es de 0,0 a 5,0 ).
} 
un $25 \%$ es media completa y el 18,7 \% básica primaria completa.

El 84,3\% de los estudiantes recibieron formación básica y media en el contexto urbano y el $87,5 \%$ en instituciones públicas. El 90,6 \% tiene computador en casa y cuenta con servicio de internet.

El $84,3 \%$ y el $93,7 \%$ califican su habilidad para leer y escribir, respectivamente, entre regular y buena. El 81,2\% precisa que tuvieron que ajustar sus prácticas de lectura y escritura al ingresar a la universidad, como se lee a continuación:

Me tocó cambiar debido a que en la secundaria no leía completos los libros académicos, solo lo que era de mi interés o le que pedían de tarea, y en la universidad me tocó empezar a leer todo aquello que fuera necesario. (e. 15.c)

[...] antes de entrar al programa pensaba que tenía buen nivel de lectura pero son insuficientes para manejar con facilidad las lecturas técnicas que se ven. (e. 9. p)

Cuando llegué a la universidad, las herramientas para leer y escribir no eran buenas y me ha tocado duro. (e. 33. s)

En el colegio en el que estuve en primaria y bachillerato, mis profesores fueron exigentes, nos daban guías y libros suficientes como apoyo para mejorar la lectura y la escritura. (e. 21. c)

El 93,7\%, de los estudiantes dicen que los trabajos universitarios los realizan a partir de la consulta de documentos impresos y de la red y el $68,7 \%$, que hacen un uso adecuado de fuentes (lo que se contradice con los resultados de la prueba en la citación de fuentes) y el $100 \%$ expresa que las orientaciones de los profesores contribuyen a fortalecer sus competencias para escribir. El 50 \% lee revistas y en particular disponibles online, el $65,6 \%$ de los estudiantes no lee periódicos.

De acuerdo con lo anterior, se identifica que los tres grupos tienen características similares. Sin embargo, una diferencia importante se precisa en los estímulos recibidos durante la infancia para el desarrollo de hábitos de lectura y escritura. El $66,5 \%$ de los estudiantes de séptimo semestre dicen que recibieron estímulos para lectura y para la escritura:

Me estimularon con la lectura de cuentos, las planas y los dictados. (e. 24. s)

En la casa me compraban libros, lo que me mejoró la ortografía. (e. 29. s)

Mi mamá me leía toda clase de cuentos, era muy dedicada, eso creo, lo que me ayudó con mis posibilidades para leer y escribir mejor. (e. 32. s)

En relación con los resultados de cuarto semestre, el $68,75 \%$ de los estudiantes manifiesta que no recibieron ningún tipo de estímulo:

No recibí ningún estímulo para la lectura y la escritura porque mis papás trabajaban la mayor parte del tiempo y me cuidaba era mi abuela y no le interesaba el tema, (e.16.c)

[...] de estímulos, ninguno; en mi casa no me compraban cuentos ni nada que me ayudara a motivarme. (e. 18. c)

Respecto a primer semestre, el 66,6 \% indica que recibieron estímulos en el desarrollo de la escritura: "Mi hermano, que es mayor, me ayudaba con las tareas de escribir y me corregía cómo escribía" (e.3.p); "Mi mamá me ponía muchas planas para mejorar la letra". (e. 11. p).

\section{Conclusiones}

Los niveles de lectura y escritura con los que ingresan los estudiantes son bajos, lo que se identifica en los puntajes de las Pruebas Saber 11 y se ratifica con la conducta de entrada procesos comunicativos que se aplica en el Programa de Permanencia y Retención Estudiantil de la Universidad de los Llanos (PREU). Estos resultados los ubica en el perfil de riesgo por razones académicas. 
Según lo anterior, el grupo de cuarto semestre presenta los niveles más bajos en la Prueba Saber 11 , y en los resultados de la prueba aplicada tiene similar comportamiento con el grupo de primer semestre en los requerimientos para la lectura crítica y la argumentación, y una leve mejoría en el resumen. Lo que implica que, luego de cuatro semestres de formación en el programa, se logra compensar los 6,7 puntos de desventaja con los que ingresa dicho grupo.

El grupo de séptimo está 2,8 puntos por encima del de primer semestre en los resultados de la Prueba Saber 11 y 9,5 por encima del de cuarto. Los resultados de la prueba aplicada para la investigación están por encima en 0,6 puntos en la elaboración del resumen, 0,9 en lectura crítica y 1,0 en argumentación, frente al primer semestre y 0,4 , 0,9 y 0,9 puntos, respectivamente, en relación con el grupo de cuarto; esto supone que es el grupo del que se espera un mejor desempeño, pero no alcanza el nivel deseado para un estudiante universitario, pese a que logra el 50 \% más en argumentación frente a los otros dos grupos y alcanza 1,7 de promedio en la lectura critica.

En ese sentido, los estudiantes que no desarrollan el nivel deseado en el requerimiento de lectura crítica no logran interpretar, inferir, analizar, explicar el sentido global de los textos y asumir una postura propia sobre los textos abordados en su formación como maestros para la infancia, lo que tiene una relación directamente proporcional con el pensamiento crítico y con el nivel de aprendizaje y desempeño académico.

Los resultados de la investigación indican que el nivel con el que ingresa el estudiante no es mejorado sustancialmente durante la formación que se da en el programa, pese a que los estudiantes sostienen que reciben de sus profesores retroalimentación en sus trabajos que les permite fortalecer sus habilidades. Sin embargo, al pedirles que aclaren las orientaciones que les dan, se observa que están dadas a la estructura de los escritos, sin aclarar cómo pasar de la narración hacia el desarrollo de argumentos, contrargumentos y asumir posiciones frente a los autores abordados, cómo construir tesis, desarrollarlas y sostener los argumentos.

Por otro lado, el 62,4\% de los estudiantes tienen madres con un nivel de formación entre básica primaria y media (aquí, se tiene en cuenta quiénes reportan formación incompleta en básica primaria y media), un 21,8 \% tiene formación técnica, y el 14,6\% formación profesional. Al respecto, los estudios muestran una relación proporcional entre el nivel educativo de la madre y el desempeño académico de los hijos. No obstante, Ilama la atención que los estudiantes con menor desempeño en la prueba aplicada al principio de la investigación tienen madres con niveles mayores de formación que arroja el grupo, más si se tiene en cuenta que "el ingreso y permanencia en la universidad están mediatizados por la capacidad de lectura y de escritura del estudiante, y esta, a su vez, está mediatizada por condiciones socioeconómicas y culturales de la familia" (Londoño, 2013, p. 67).

Los estudiantes que reportaron experiencias en la infancia de estimulación para la lectura y la escritura son quienes presentan mejores desempeños en la prueba aplicada en la investigación. Lo que indica que para los participantes en la investigación hay una relación entre los procesos de estimulación recibidos a temprana edad en lo que respecta a la lectura y la escritura y su impacto en la adultez. Este aspecto debe ser investigado en profundidad en futuros estudios.

La mayoría de los estudiantes en primer semestre escriben igual a como hablan, tienen problemas de cohesión y coherencia, y abuso de adjetivos. Tienen claridad sobre la dimensión de la lectura crítica pero no tienen herramientas para construir tesis, argumentos para desarrollarlos y sostenerlos.

En general, al identificar el bajo nivel de lectura y escritura de los estudiantes, se puede concluir que tienen impedimentos para apropiar lo que expresan los textos que se les sugieren en los diferentes cursos para formarse como maestros, con 
discursos y posturas pedagógicas que les permitan desempeñarse de manera adecuada en el mundo laboral, con capacidad de lectura que les facilite seguir actualizándose, además con habilidad para escribir de forma coherente y cohesiva, con estilo propio y agradable.

Los estudiantes alcanzan en cuarto y séptimo semestres un nivel satisfactorio en el desarrollo del resumen. No obstante, con el trabajo en macrorreglas, se puede mejorar el desempeño de aquellos que ingresan a la universidad, especialmente si se les presentan elementos analíticos que afiancen los procesos comprensivos y productivos del texto. Estos argumentos pueden provenir de la lingüística textual, el análisis crítico del discurso y la argumentación pragmadialéctica, que son opciones viables y pertinentes en los propósitos académicos. El enfoque desde el cual se formula una propuesta se validará en la segunda fase del proyecto. Esto es crucial si se tiene en cuenta que para Paula Carlino (2005), las habilidades de lectura y escritura no son separables y aprendidas de forma definitiva, sino que deben trabajarse, por ejemplo, en la universidad, en el marco de una matriz disciplinar respecto de la naturaleza de lo que debe ser aprendido (conceptos y discursos) por los futuros profesionales.

Finalmente, es pertinente indicar que, si bien no fue un propósito de esta fase investigativa el preguntarse a profundidad por las prácticas pedagógicas de los profesores que desarrollan cursos en el programa de Pedagogía Infantil de la Universidad de los Llanos, en el intento de avanzar en una propuesta para favorecer los niveles de lectura y escritura en los estudiantes se ha logrado identificar que debe posicionarse como un eje transversal y no como actividad aislada. Así las cosas, es necesario que se asuma como una política del programa, para lo cual es indispensable que los profesores como especialistas de la disciplina se hagan cargo de la tarea de lectura y escritura con los estudiantes, puesto que además de conocer las convenciones propias de sus cursos, están familiarizados con el contenido que se espera dominen (Bailey y Vardi, 1999).

\section{Reconocimientos}

Este artículo es producto de la investigación titulada "Niveles de lectura y escritura en estudiantes del programa de Pedagogía Infantil de la Universidad de los Llanos. Primera fase", del grupo Infancias, Educación y Contexto de la Universidad de los Llanos, concluida el 31 de mayo de 2019, investigación financiada por la institución.

\section{Referencias bibliográficas}

Almeida, A., Matías, R. y Fernández, J. (2010). Cómo escribir el resumen de un artículo científico en inglés. Revista Habanera de Ciencias Médicas, 9(3), 403-414.

American Psychological Association (APA) (2014). Normas para citar información bibliográfica. Nueva York: Editorial.

Bailey, J. y Vardi, I. (1999). Iterative feedback: impacts on student writing. Ponencia presentada en la Conferencia Internacional Anual de la Sociedad Australiana para la Investigación y Desarrollo de la Educación Superior. Melbourne.

Beaugrande, R. y Dressler, W. (1997). Introducción a la lingüística del texto. Barcelona: Ariel Lingüística.

Betancourt, R. y Frías, L. (2015). Argumentative skills of law students in the framework of the Saber-Pro tests. Civilizar Ciencias Sociales y Humanas, 15(28), 213-228.

Borreguero, M. y López, A. (2011). Marcadores discursivos, valores semánticos y articulación informativa del texto: el peligro del enfoque lexicocentrista. En H. Aschenberg (ed.), Marcadores del discurso: de la descripción a la definición (pp. 169-212). Madrid: Iberoamericana.

Braslavsky, B. (2005). Enseñar a entender lo que se lee: la alfabetización en la familia y en la escuela. Buenos Aires: Fondo de Cultura Económica.

Briz, A. y Pons, S. (2010). Unidades, marcadores discursivos y posición. En Ó. Loureda y E. Acín (coords.), Los estudios sobre marcadores del discurso, hoy (pp. 327-358). Madrid: Arco/Libros. 
Calderón, A. y Quijano, J. (2010). Características de comprensión lectora en estudiantes universitarios. Estudios Socio-Jurídicos, 12(1), 337-364.

Camps, A. et al. (2011). La enseñanza de la ortografía. Barcelona: Graó.

Cardona, P. y Londoño, D. (2016). El sentido de la lectura crítica en contexto. Katharsis, (22), 375-401.

Carlino, P. (2005). Escribir, leer y aprender en la universidad. México: Fondo de Cultura Económica.

Casas, R. (2015). El dequeísmo: ¿Un cambio en progreso de la sintaxis castellana? Letras, 86(124), 289-310.

Cassany, D. (1995). La cocina de la escritura. Barcelona: Anagrama.

Cassany, D. (1999). La composición escrita en E/LE. Monográficos Marco ELE: Revista de Didáctica del Español como Lengua Extranjera, 9, 47-66.

Cassany, D. (2004). Explorando las necesidades actuales de comprensión. Aproximación a la comprensión crítica. Tomado del proyecto de investigación "La comprensión receptiva crítica en estudiantes universitarios y de bachillerato: análisis y propuesta didáctica". Código HUM2004-03772. Barcelona: Universitat Pompeu Fabra.

Cassany, D. (2006). Tras las líneas. Sobre lectura contemporánea. Barcelona: Anagrama.

Castañeda, L. S. y Henao, J. I. (2005). La lingüística textual y la cultura escrita en la universidad. Zona Próxima, (6), 12-31.

Cuetos, F., Molina, M., Suárez, P. y Llenderrozas, M. (2017). Validación del test para la detección temprana de las dificultades en el aprendizaje de la lectura y escritura. Pediatría Atención Primaria, 19(75), 241-246.

Denzin, N. y Lincoln, Y. (2012). Manual de investigación cualitativa. Barcelona: Gedisa.

Eco, U. (1985). Obra abierta. Madrid: Alcaná Libros.

Espinosa, P. (2017). Aportes de Mijaíl Bajtín a la crítica dialógica. Revista Aisthesis, (27), 37-44.

Ferreiro, E. (2003). La escuela no forma buenos lectores. Buenos Aires: La Nación.

Gastel, B. y Day, R. (2016). How to write and publish a scientific paper. Nueva York: ABC-CLIO.
Henao, J. I. y Castañeda, L. S. (2002). El papel del lenguaje en la apropiación del conocimiento. Bogotá: ICFES.

Henao, J. y Toro, L. (2008). Cultura escrita y educación superior: el caso de la Universidad de Antioquia y la Universidad de Medellín. En E. Narváez, Los desafíos de la lectura y la escritura en la educación superior: caminos posibles (pp. 53-74). Cali: Universidad Autónoma de Occidente.

Henao, J., Londoño, D., Frías, L. y Castañeda, L. (2011). Niveles de literacidad de los estudiantes de psicología de la institución universitaria de Envigado. Zona Próxima: Revista del Instituto de Estudios Superiores en Educación, (15), 54-77.

Huguet, J., Gaya, J., Rodríguez, O., Breda, A. y Palou, J. (2018). El estilo de la comunicación científica. Actas Urológicas Españolas, 42(9), 551-556 DOI: 10.1016/j.acuro.2018.02.013.

Hussein, A. (2015). The use of triangulation in social sciences research: Can qualitative and quantitative methods be combined? Journal of Comparative Social Work, 4(1), 1-12.

Hyland, K. (2015). Teaching and researching writing. Nueva York: Routledge.

Isaza, J. F. (27 de junio de 2012). Lecto-escritura. Recuperado de https://www.elespectador.com/opinion/ lecto-escritura-columna-355796/

Lewin, K. (1973). Action research and minority problems. En K. Lewin y G. Lewin (eds.), Resolving social conflicts: selected papers on group dynamics (pp. 201-216). Londres: Souvenir Press.

Londoño, D. A. (2013). Análisis sociolingüístico de los niveles de literacidad en jóvenes de la Institución Universitaria de Envigado. [Tesis doctoral]. Centro de Estudios Avanzados en Niñez y Juventud-Universidad de Manizales-Cinde. Manizales.

Londoño, D. A. (2015). De la lectura y la escritura a la literacidad: una revisión del estado del arte. Anagramas: Rumbos y Sentidos de la Comunicación, 14(26), 197-220.

Londoño, D. A. (2016). Análisis sociolingüístico de literacidad en jóvenes de la Institución Universitaria de Envigado. Revista Lasallista de Investigación, 13(1), 49-64. 
Londoño, D. A. y Ospina, M. (2018). Comprensión y producción de textos escritos en instituciones de educación superior. Trilogía Ciencia Tecnología Sociedad, 10(18), 183-202.

Londoño, D. A., Henao, J. I., Frías, L. L. y Marín, G. S. (2010). El análisis del discurso y la formación de lectores críticos, autónomos y democráticos. Bucaramanga: Fundación El Libro Total.

López, G. S. y Arciniegas, E. (2003). El uso de estrategias metacognitivas en la comprensión de textos escritos. Lenguaje, (31), 118-141.

Louwerse, M. (2004). Un modelo conciso de cohesión en el texto y coherencia en la comprensión. Revista Signos, 37(56), 41-58.

Lozano, M., Hernández, G., Ortiz, E., \& Chacón, L. (2015). Prácticas de escritura. En B. González y A. Salazar-Sierra (Eds.), Formación inicial en lectura y escritura en la universidad: De la educación media al desempeño académico en la educación superior (pp. 135-181). Bogotá: Editorial Pontificia Universidad Javeriana.

Mayor, B. (2006). La citación en la comunicación médica escrita (inglés-español): funciones y tipos. Lebende Sprachen, 51(2), 72-78.

McNamara, D. (2004). Aprender del texto: efectos de la estructura textual y las estrategias del lector. Revista Signos, 37(55), 19-30.

Moreno, V. (2012). De la argumentación pragmadialéctica como competencia investigativa en los estudiantes de derecho de la Institución Universitaria de Envigado. Ratio Juris, 7(15), 123-148.

Moyano, E. y Giudice, J. (2016). Un programa de lectura y escritura universitario: Lineamientos teóricos, características y resultados de aplicación. Revista Grafía-Cuaderno de trabajo de los profesores de la Facultad de Ciencias Humanas, 13(1), 33-59.

Newell, G., Bloome, D. y Hirvela, A. (2015). Teaching and learning argumentative writing in high school English language arts classrooms. Nueva York: Routledge.

Ossa, M. (2003). Pautas para citar textos y hacer listas de referencias según las normas de la American
Psichological Association (APA). Revista EMA, 8(3), 335-349.

Pinker, S. (2011). Si yo gobernara el mundo. Revista El Malpensante, 126, Recuperado de https://www.elmalpensante.com/articulo/2236/ si_yo_gobernara_el_mundo

Quitián, S. (2017). Oralidad y escritura: campos de investigación en pedagogía de la lengua materna. Revista Praxis y Saber, 8(16), 133-153.

Rodríguez, Ó. (2005). La triangulación como estrategia de investigación en ciencias sociales. Tribuna de Debate, 31(2). Recuperado de https://www.madrimasd.org/revista/revista31/tribuna/tribuna2.asp.

Salazar, A., Sevilla, O., González, B., Mendoza, C., Echeverri, A., Quecán, D., ..., Lozano, M. (2015). Lectura y escritura en la universidad: contribución para reconstruir una historia. Magis, Revista Internacional de Investigación en Educación, 8(16), 51-70.

Sánchez, A. (2015). Análisis lingüístico de la citación en artículos de ciencias sociales y humanas. Revista Lasallista de Investigación, 12(1), 99-124. DOI: 10.22507/rli.v12n1a10.

Serradilla, A. (2017). El avance del dequeísmo en el siglo XIX: factores desencadenantes. Revista de Filología Española, 97(1), 145-173.

Serrano, S. (2014). La lectura, la escritura y el pensamiento. Función epistémica e implicaciones pedagógicas. Lenguaje, 42(1), 97-122.

Stubbs, M. (2014). Language and Literacy (RLE Linguistics C: Applied Linguistics): The Sociolinguistics of Reading and Writing. Nueva York: Routledge.

Torres, P. y Londoño, D. A. (2016). El sentido de la lectura crítica en contexto. Katharsis, (22), 375-401. Recuperado de https://dialnet.unirioja.es/servlet/ articulo?codigo $=5888105$

Van Dijk, T. (1991). La ciencia del texto. Barcelona: Paidós.

Van Dijk, T. (1999). Ideología: una aproximación multidisciplinaria. Barcelona: Gedisa.

Van Eemeren, F. y Grootendorst, R. (2006). Argumentación, comunicación y falacias: una perspectiva pragma-dialéctica. Santiago de Chile: Universidad Católica de Chile. 
Van Eemeren, F., Garssen, B., Krabbe, E., Snoeck, A. V. y Wagemans, J. H. (2014). Handbook of argumentation theory. Ámsterdam: Springer.

Wallace, M. y Wray, A. (2016). Critical reading and writing for postgraduates. Londres: Sage.
Zavala, I. (1989). Dialogía, voces, enunciados: Bajtín y su círculo. En G. Reyes (coord.), Teorías literarias en la actualidad (pp. 79-128). Buenos Aires: El Arquero.

\section{(c) (1) $\$(0)$}

